\title{
Vacíos para la Explotación Legal de Huevos de la Tortuga Lora (Lepidochelys olivacea) en el Refugio Nacional de Vida Silvestre Ostional, Costa Rica
}

\author{
Lucía Isabel López y José Manuel Mora²
}

Resumen. La tortuga marina Lepidochelys olivacea anida solitaria o dispersa, en arribada y en condición mixta. Las arribadas son eventos de anidación masiva y sincronizada que solo ocurren en algunas playas, una de las más importantes del mundo es Ostional en el noroeste de Costa Rica. Esta playa es parte del Refugio Nacional de Vida Silvestre de Ostional (RNVSO) que fue creado para proteger a las tortugas marinas en anidación y regular la extracción de huevos. A partir de ahí se emitió un decreto que permite la explotación legal de los huevos de la tortuga lora por la comunidad de Ostional mediante un aprovechamiento que es manejado por la Asociación de Desarrollo Integral de Ostional (ADIO). Este aprovechamiento es un gran ejemplo del uso de la biodiversidad por las comunidades nativas según los lineamientos de la CBD (Convenio sobre Diversidad Biológica). No obstante, hay varios vacíos para la explotación de huevos y la protección de la tortuga lora. En este trabajo analizamos varios factores relacionados con las tortugas marinas en Ostional y la explotación de sus huevos. Tratamos de identificar los vacíos principales que existen para que esta explotación tenga los resultados pretendidos con el decreto de aprovechamiento y con la creación del RNVSO. El primer vacío es la falta de un plan general de manejo oficializado para el refugio. La efectividad de manejo de un área protegida depende en primer lugar de su plan de manejo. Asociado a este punto está uno de los vacíos principales del refugio, su definición como tal. Al momento no se sabe si el refugio es estatal o mixto. Esa falta de definición crea gran inestabilidad al refugio y a la comunidad de Ostional. Otro vacío es la falta de información del estado poblacional de la tortuga lora. Aparentemente, las metodologías usadas no han sido adecuadas para el monitoreo de la población. Aunque se argumenta que la población que anida ha aumentado, no hay publicaciones científicas que respalden este argumento. Tampoco hay respaldo comprobado que la explotación de huevos beneficie directamente a la población de la tortuga lora que anida en Ostional. Tampoco se han investigado los aspectos relacionados a posibles zoonosis por las interacciones con las tortugas ni debidas al consumo de huevos. Aunque el ecoturismo es una alternativa económica para Ostional, tiene el vacío de un manejo adecuado. En todos estos aspectos el MINAE (Ministerio del Ambiente y Energía), como ente responsable de la vida silvestre del país, debe tomar el liderazgo que le corresponde. Sus acciones deben asociarse con la ADIO y las instituciones responsables para garantizar el bienestar de la comunidad de Ostional y la conservación de la tortuga lora.

Palabras clave: Conservación biológica, manejo de vida silvestre, playas de anidación, tortugas marinas.

\section{Gaps in the Legal Exploitation of Kemp's Ridley (Lepidochelys olivacea) Eggs at Ostional National Wildlife Refuge, Costa Rica}

Abstract. The Olive Ridley sea turtle nests lonely, in arribada and in mixed condition. The arribadas are massive and synchronized events that only occur on some nesting beaches, one of the most important in the world is Ostional in northwestern Costa Rica. This beach is part of the National Wildlife Refuge Ostional (ONWL) which was created in order to protect sea turtle nesting and regulate the egg extraction. From there a decree allowing the legal exploitation of olive Ridley eggs by Ostional community members was issued. The harvest is managed by the Ostional Development Association (ADIO). This communal egg harvesting is a great example of the use of

\footnotetext{
${ }^{1}$ Bióloga y consultora ambiental, Cinco Esquinas, Carrizal, Alajuela, Costa Rica. Email luciaisa2@gmail.com

2 Instituto Internacional en Conservación y Manejo de Vida Silvestre (ICOMVIS), Universidad Nacional (UNA), Heredia, Costa Rica. Email josemora07@gmail.com
} 
biodiversity by indigenous communities along the lines of the CBD (Convention on Biological Diversity). However, there are many loopholes to exploiting eggs and the protection and conservation of the Kemp's Ridley turtle. This paper analyzes different factors related to sea turtles in the Ostional and the exploitation of their eggs. We try to identify the main gaps that exist for this operation compared to the intended results of the decree of exploitation and the creation of the ONWL. The first gap is that the ONWR does not have a formalized management plan. Management effectiveness of a protected area depends on its management plan. Associated with this point is one of the main gaps in the refuge; its definition as such. At the moment it is not known if the refuge is state owned or mixed. This uncertainty creates great instability to both the protected area and the community of Ostional. Another gap is the indeterminacy of the population status of the Kemp's Ridley turtle. Apparently the methodologies used for this purpose have not been adequate to monitor the population. Although it is argued that the population of olive Ridley nesting in Ostional has increased, there is no scientific literature to support this argument. Similarly no proven backup exits for the idea that harvesting eggs have direct benefits for the population of the olive Ridley nesting in Ostional. Also aspects of possible zoonosis due to interactions with turtles or from egg consumption have not been investigated. Although ecotourism is an economic alternative for Ostional, it lacks proper management. In all these respects the MINAE (Ministerio del Ambiente y Energía), as head of the country's wildlife patrimony, is called to take the leadership. Their actions must be associated with those of ADIO and all institutions responsible for ensuring the welfare of the community of Ostional and conservation of the Kemp's Ridley.

Key words: Biological conservation, nesting beaches, sea turtles, wildlife management.

\section{Introducción}

La tortuga lora (Lepidochelys olivacea) es la especie de tortuga marina más abundante (NMFS y USFWS 2014). La especie se distribuye en las zonas tropicales y subtropicales de todo el mundo (Eckert y Abreu Grobois 2001). No obstante, esta tortuga es considerada una especie vulnerable por la Unión Internacional para la Conservación de la Naturaleza UICN y está incluida en el apéndice I de la Convención sobre el Comercio Internacional de Especies Amenazadas de Fauna y Flora Silvestres - CITES (Eckert y Abreu Grobois 2001). Según esto, está prohibido cualquier tipo de explotación y los productos derivados de la tortuga lora.

La tortuga lora tiene tres modos de anidación: arribada, anidación dispersa o en solitario y condición mixta (Bernardo y Plotkin 2007). La forma más común de anidación es dispersa o solitaria, sin aparente sincronía entre los eventos de anidación. En algunas localidades, también puede existirr una mezcla de esta con el tercer modo de anidación (Abreu-Grobois y Plotkin 2008). El tercer modo es un comportamiento de anidación sincrónica, conocido como flota o arribada (ADIO et al. 2012), que consiste en la llegada simultánea de tortugas para desovar en una misma playa. Este comportamiento fue descrito por Hildebrand (1963) basado en un video filmado en
Rancho Nuevo, México, en 1947, de una arribada de Lepidochelys kempi (Orrego 2014).

El fenómeno de arribadas se ha registrado principalmente en Costa Rica, India y México (Valverde et al. 2010). En Costa Rica, hay dos playas de arribadas o anidación masiva localizadas en el noroeste del país. Una es Nancite, dentro del Parque Nacional Santa Rosa, y la otra en el Refugio Nacional de Vida Silvestre Ostional.

Cada año desde 1959, cientos de miles de tortugas lora llegan a un pequeño tramo de la playa de Ostional para depositar sus huevos (Cornelius et al. 1991). Las arribadas son de los mayores eventos de anidación grupal conocidos. No obstante, poco se sabe sobre los mecanismos biológicos que las producen (Orrego 2014). Este tipo de anidación masiva ha facilitado el uso de la especie por los humanos, lo que ocurre, generalmente, de manera ilegal (Valverde et al. 2012). Como consecuencia de este y otros impactos, la abundancia de las anidadas en varias de las playas de anidación más importantes ha bajado (Valverde et al. 1998, Orrego 2014).

El uso sostenible de los productos de la biodiversidad puede ser visto como una estrategia para el manejo de hábitats naturales y por lo tanto para la conservación de los ecosistemas (Becerra 2003). El uso sostenible permite la permanencia de los recursos 
López y Mora: Vacíos para la Explotación Legal de Huevos de la Tortuga Lora (Lepidochelys olivacea)

en el tiempo y se generan beneficios económicos para las comunidades locales. Estas, a su vez, reconocen el valor de los recursos y trabajan en su conservación (Becerra 2003).

La comunidad de Ostional, de unos 600 habitantes, en Santa Cruz, Guanacaste en el pacífico costarricense, ejecuta el único proyecto de explotación legal de huevos de tortuga lora. En Ostional aprovechan estos huevos para el consumo y comercialización. La organización comunitaria Asociación de Desarrollo Integral de Ostional (ADIO) se encarga de los aspectos prácticos y administrativos del proyecto. La ADIO cosecha los huevos puestos durante los primeros 2.5 días de cada arribada, que por lo general tiene una duración de 5 días. Los huevos recolectados en los primeros 2 días se comercializan. La cosecha del medio día restante es para el consumo de los miembros de la ADIO.

Para algunos científicos, el proyecto de Ostional es un caso ejemplar de aprovechamiento sostenible (García y McHugh 2005). Este ha permitido el desarrollo de la comunidad de forma directa a través de la comercialización de los huevos e indirecta mediante el turismo y la investigación asociados a las arribadas (ADIO et al. 2008). Así mismo, desde sus inicios, el proyecto cuenta con detractores que argumentan sobre el impacto negativo potencial de la explotación en las poblaciones de L. olivacea.

El aprovechamiento de los recursos naturales implica el diseño de sistemas de aprovechamiento dependientes del conocimiento tradicional y científico de la biología de las especies y sus relaciones ecológicas (Becerra 2003). Se deben incorporar sistemas de monitoreo para determinar el desempeño del manejo y el estado de los recursos a lo largo del tiempo. El conocimiento de los parámetros poblacionales es vital para manejar o explotar una especie (Mora 2003). El manejo adaptativo es muy útil en la evaluación y monitoreo porque enfatiza el continuo registro de datos y su análisis para usarlos en las decisiones de manejo (Mora 2003).

Desafortunadamente, la comunidad científica no tiene estudios contundentes acerca del impacto de la explotación de huevos de tortuga en Ostional. Desde la década de 1970, la Universidad de Costa Rica (UCR) ha monitoreado las poblaciones de la tortuga lora en playa Ostional. Desde el establecimiento del proyecto de aprovechamiento, la UCR ha trabajado para detectar cambios en la dinámica poblacional que adviertan sobre el impacto de la explotación. Otros investigadores e instituciones se han sumado a esta tarea. No obstante, los datos generados son inconclusos y las opiniones acerca del impacto en las poblaciones de la tortuga lora varían entre las organizaciones involucradas y los estudios realizados (NMFS y USFWS 2014, Orrego 2014).

En este reporte analizamos los beneficios, fortalezas y aciertos, así como los aspectos negativos y los argumentos que hay en contra del programa de aprovechamiento y comercialización de los huevos de la tortuga lora en Ostional. Este análisis tiene como objetivo exponer los vacíos existentes para que la explotación de huevos de la tortuga lora en Ostional tenga un sustento contundente en lo científico, legal y comunitario.

\section{Metodología}

Área de estudio. El Refugio Nacional de Vida Silvestre Ostional (RNVSO) está situado en la costa oeste de la Península de Nicoya en el pacífico norte de Costa Rica. El refugio fue creado por ley el 17 de noviembre de 1983 con el propósito de proteger los sitios de anidación de la tortuga lora. EI RNVSO es considerado el principal sitio de anidación en el mundo para la tortuga lora debido al tamaño y la frecuencia de las arribadas (SINAC 2014). Esta área protegida tiene 320 ha terrestres y 8,000 marítimas. La franja costera tiene $200 \mathrm{~m}$ de ancho y $16 \mathrm{~km}$ de largo que incluye el área de arribadas de $7 \mathrm{~km}$ de extensión. No obstante, la mayor parte de las arribadas tienen lugar en una tramo de solo $1.0 \mathrm{~km}$ de extensión, denominado playa de anidación principal (ADIO et al. 2012).

Recopilación de la información. En mayo de 2014 dedicamos 33 días/hombre para conocer en el sitio las opiniones, estrategias, actividades, supuestos y beneficios del proyecto de aprovechamiento de los huevos de la tortuga lora en Ostional. Entrevistamos a líderes comunales, vecinos y representantes de diferentes organizaciones involucradas en la investigación y en el manejo del proyecto. En julio de 2015, hicimos las mismas actividades con aproximadamente 20 días/hombre de trabajo. Para estas actividades contamos con el apoyo de los estudiantes de las promociones XXV y XXVI del ICOMVIS (Instituto Internacional en Conservación y Manejo de Vida Silvestre) de la UNA (Universidad Nacional) de Costa Rica.

Entrevistamos a personas involucradas con el proyecto tales como los personeros de la UCR, el 
MINAE (Ministerio del Ambiente y Energía), el RNVSO, la ADIO y algunos habitantes del lugar. Efectuamos caminatas de reconocimiento en la comunidad y la zona de anidación de las tortugas marinas. Hicimos una búsqueda de la literatura científica y en general relacionada con el proyecto de Ostional.

\section{Resultados y Discusión}

La explotación de los huevos de la tortuga lora por la comunidad de Ostional empezó en 1959 (Chaves 2007), mismo año que supuestamente empezaron las arribadas en el sitio. A partir de 1970 se empezaron a monitorear las arribadas e inició la investigación de su población (Cornelius et al. 1991). Desde los 80 surgió la idea de un proyecto de aprovechamiento de los huevos de la tortuga lora en Ostional. En 1987 inició la cosecha regulada de los huevos por la comunidad (Cornelius et al. 1991). Desde ese momento, se han aprovechado miles de huevos en casi todas las arribadas. Unas excepciones se han dado por conflictos legales para el permiso de operación o la saturación del mercado de huevos de tortuga (ADIO et al. 2012).

Anidación de la tortuga lora y justificación de
aprovechamiento de sus huevos. Debido a la alta densidad de tortugas que llegan a desovar a la playa de Ostional, la mayoría de los nidos al inicio de la arribada son destruidos por las hembras que llegan después en la misma arribada (Cornelius et al. 1991). Esta destrucción aumenta la concentración de hongos y bacterias, lo que aumenta la mortalidad embrionaria (NMFS y USFWS 2014). Lo anterior se ha visto como una oportunidad para aprovechar los huevos de los primeros días de la arribada que de otra manera serían destruidos. La remoción de los huevos para venderlos, unos cuatro millones por año, favorece las condiciones para el desarrollo y eclosión de los huevos que permanecen en la playa (Cornelius y Robinson 1985, Cornelius et al. 2007, Chaves 2007). Esto se debe a la disminución de la carga bacteriana, la oxigenación de la arena, la disminución de la densidad de nidos y por lo tanto el aumento en la disponibilidad de espacio en la playa para anidar (NMFS y USFWS 2014). No obstante, estos aspectos no han sido demostrados científicamente (Orrego 2014).

El éxito en el desarrollo de los huevos de tortuga lora es naturalmente bajo (Valverde et al. 2010) y en una arribada se pierde hasta el $50 \%$ de los huevos debido a la alta densidad de nidos. A esto se suma la alta mortalidad por depredación de huevos y crías (Cornelius et al. 1991) y por la contaminación de los huevos con microorganismos (Madden et al. 2008). La baja tasa de eclosión en la playa principal de anidación (30\%), es uno de los principales argumentos utilizados para justificar el aprovechamiento de un porcentaje de los huevos (Valverde et al. 2012). Así, el aprovechamiento podría constituir un factor beneficioso para las tortugas al disminuir la cantidad de materia orgánica que es el sustrato para los microorganismos patógenos (NMFS y USFWS 2014).

Se cree que el comportamiento de arribada es para saturar a los depredadores y así incrementar la posibilidad de supervivencia de las crías (Pritchard y Plotkin 1995). Se ha argumentado que esta saturación de depredadores posiblemente se ve afectada en Ostional debido a la intervención humana. No obstante, los alrededores de la playa Ostional están muy degradados y la cantidad de depredadores debe ser mucho menor de lo natural para el sitio. A la vez, los miembros de la ADIO ayudan a cuidar las crías y disminuir la depredación que ocurre principalmente por animales domésticos $y$ especies comunes $y$ abundantes como zopilotes y mapaches. No obstante, se han observado otros depredadores no tan comunes en el sitio como el garzón Mycteria americana (Burger y Gochfeld 2013) y la pitón americana Loxocemus bicolor (Mora y Robinson 1984).

El aprovechamiento de los huevos de la tortuga lora en Ostional se hace bajo planes quinquenales (periodo 2007-2011, ADIO et al. 2008 y periodo 20122016, ADIO et al. 2012). Estos planes han sido elaborados por los grupos involucrados en la explotación y guían el aprovechamiento de los huevos de las tortugas en Ostional (ADIO et al. 2012). Sin embargo, el RNVSO, como área protegida, no tiene un plan de manejo, lo cual constituye un vacío serio para la efectividad de manejo del refugio.

El RNVSO tiene ya 32 años de establecido, pero no tiene un plan de manejo oficializado. Un plan de manejo para Ostional debe contener análisis de la biología de la especie, el conocimiento comunitario, la cultura del lugar e incluir programas de conservación, protección, manejo, restauración y gestión, que se ajusten al entorno social, histórico, cultural y biológico del área. Dicho plan debería ser el eje rector para la regulación y establecimiento de todas las actividades, las acciones y los lineamientos básicos para el manejo y administración del refugio, incluidas las bases para el aprovechamiento de los huevos de tortuga. 
Los planes quinquenales incluyen actividades de manejo como la quema de madera y siembra de árboles, además, la remoción de la materia vegetal (troncos, hierba y vegetación rastrera) que puede interferir con el movimiento de las tortugas y las crías en la playa (ADIO et al. 2008, ADIO et al. 2012). La ADIO vigila para evitar la extracción ilegal de los huevos por personas ajenas al proyecto. También trasladan nidadas de tortugas a zonas con más humedad 0 sombra cuando las temperaturas sobrepasan el ámbito de tolerancia térmica para el desarrollo embrionario (Chaves 2007). No obstante, no hay pautas de manejo establecidas bajo un plan de manejo general del área basado en las características poblacionales de la especie y las condiciones del ecosistema costero y su entorno ecológico y social.

Hay recomendaciones sobre áreas de no extracción en algunos sectores de la playa, pero no se han seguido. Esto supone una contradicción en la toma de decisiones para el manejo pues este debe estar basado en el conocimiento científico, las opiniones de los expertos y ser regulado por las autoridades del estado (Orrego 2014).

La extracción de los huevos en Ostional está en función de la demanda del mercado. Esta extracción es un bajo porcentaje de la totalidad de los huevos puestos en una arribada por las tortugas. Ello significa que la extracción no afectaría de manera significativa a la población de la tortuga lora en Ostional (Cornelius y Robinson 1985; Cornelius et al. 1991; Chaves y Morera 2003, ADIO et al. 2012).

\section{Implicaciones del consumo de huevos para la} salud humana. Existe cierta información básica sobre los atributos alimenticios de los huevos de tortuga (Castro-González y Pérez-Gil 2011). Sin embargo, existe un vacío en aspectos relacionados a las posibles zoonosis y sus repercusiones en la salud pública. El consumo de huevos de tortugas puede tener efectos adversos en la salud humana, ya que pueden presentar y transmitir bacterias, parásitos y contaminantes. La comunidad de Ostional no cuenta con los protocolos de higiene apropiados durante la manipulación de los huevos, previo a su venta. Además, el contacto directo con las tortugas marinas que tiene la comunidad durante las arribadas y el cuidado de crías, podría causar problemas de salud.

Varias bacterias han sido detectadas en los huevos de tortuga (Aguirre et al. 2006). La salmonelosis es la enfermedad zoonótica más conocida que transmiten los reptiles. Ocurre al comer productos crudos contaminados con Salmonella o por contacto directo (Santoro et al. 2008). También, ciertas especies de Mycobacterium pueden transferirse por contacto directo, inhalación o por contacto con las mucosas, aunque no se puede descartar que se transmita al hombre por consumo. Aunque la Chlamydophila es una zoonosis asociada a mamíferos y aves, se reportó un caso con tortugas marinas en cautiverio.

La leptospirosis es la zoonosis de mayor distribución global y ha sido clasificada como una enfermedad reemergente por la Organización Mundial de Salud (WHO). Es causada por más de 250 serotipos de espiroquetas del género Leptospira. Los mamíferos son los principales hospederos, sin embargo, se cree que las tortugas marinas pueden actuar como reservorios de algunos serotipos. Se necesitan más estudios sobre el potencial zoonótico de esta enfermedad (Aguirre et al. 2006, Warwick et al. 2013).

Una de las zoonosis más peligrosas es el de Vibrio mimicus que produce una toxina similar a la del cólera. Entre 1991 y 1994, en Costa Rica se reportaron 33 casos de V. mimicus. De estos, 11 habían comido huevos de tortuga una semana antes de presentar los síntomas de diarrea, vómito y deshidratación extrema (Campos et al. 1996). El V. mimicus se reproduce en la superficie externa de la cáscara del huevo de tortuga (Pereira-Zamora et al. 2007).

Existen varios casos documentados sobre protozoarios parásitos en varias poblaciones de tortugas marinas (Kinne 1985, Aguirre et al. 2006). Se han reportado casos de Entamoeba invadens y $E$. histolitica en tortugas en cautiverio. No se puede descartar la posibilidad de que estos parásitos puedan ser transferidos a humanos.

Se ha documentado que hay cadmio, mercurio y plomo en huevos de tortugas y en tortugas recién nacidas. Las concentraciones encontradas de estos metales causan toxicidad en otros vertebrados (Aguirre et al. 2006). Su ingesta puede incrementar el riesgo de osteoporosis, causar disfunción renal, problemas cardiovasculares y de inmunidad, infertilidad y pérdida de la visión (Aguirre et al. 2006).

Existen otros problemas de salud potenciales de los cuales no existe suficiente información. Tampoco hay estudios sobre las enfermedades que se pueden transmitir de las personas a los animales (zooantroponosis). Los habitantes de Ostional podrían estar transfiriendo enfermedades a las crías de 
tortugas o a los adultos al entrar en contacto con ellos. Al mismo tiempo, si existe contacto de una persona con una tortuga enferma y después se manipula a otro individuo sano, las personas podrían contagiar accidentalmente a otros individuos, lo cual podría resultar en una posible epizootia (Eckert et al. 2000).

Aspectos institucionales del proyecto de aprovechamiento. EI RNVSO se creó para controlar la extracción de huevos de tortuga mediante el ordenamiento de esta actividad. Para ello, la comunidad de Ostional sería la única autorizada para ejecutar el aprovechamiento a través de la ADIO.

La administración del RNVSO corresponde al MINAE por ley. No obstante, dada la naturaleza del sitio y la existencia de instituciones y organizaciones relacionadas, se constituyó la CIMACO (Comisión Interinstitucional de Manejo Compartido de Ostional). Esta comisión se encarga del manejo del área protegida y está conformada por representantes de INCOPESCA (Instituto Costarricense de Pesca y Acuicultura), MINAE, ADIO, AGLO (Asociación Guías Locales), UCR y las comunidades de Nosara y Guiones. En el 2005 se construyeron las instalaciones del SINAC-MINAE, pero la vigilancia y control en el RNVSO son pocos debido a la falta de personal y de presupuesto. Al 2015, el refugio cuenta únicamente con un administrador y tres guardaparques.

Actualmente, se busca hacer excepciones e incluso modificaciones a la ley para que se permita mayor apertura para el manejo del área. Con la creación del RNVSO, el terreno que este abarca pasó a ser patrimonio nacional y propiedad del estado, por lo que unos 300 habitantes que viven dentro de los límites geográficos del área se encuentran en una situación muy precaria de tenencia de la tierra, pese a que muchos de ellos ya se encontraban ahí antes de la creación del refugio. Las personas que poseen viviendas dentro del área del RNVSO no tienen títulos de propiedad y por lo tanto están en riesgo de ser desalojados (Asamblea Legislativa de la República de Costa Rica 2009). Esta situación puede implicar el desalojo de todas estas personas, lo que crearía un descontento generalizado en la comunidad. Si por el contrario, el Estado decide no desalojar a estas personas, el refugio incumple con los estatutos de conservación establecidos inicialmente al decretarse la zona como área protegida.

Debido al voto de desalojo por parte de la Sala Constitucional, que da un plazo para que la comunidad de Ostional deje el área del refugio, se creó el proyecto de ley TECOCOS (Territorios Costeros Comunitarios). Dicho proyecto de ley aboca la permanencia de las comunidades locales que históricamente han habitado y conservado los territorios costeros, esto con el fin de garantizar la preservación de la cultura, las costumbres y las tradiciones de estas comunidades y el mejoramiento de su calidad de vida en plena armonía con la protección del ambiente y los ecosistemas naturales.

Un vacío enorme es la definición del tipo de refugio que es Ostional pues no existe ninguna ley, decreto o reglamento en donde esté definido (Salazar y Rojas 2012). Existen dos puntos de vista sobre este asunto, por un lado se dice que se trata de un refugio estatal, mientras que también se dice que es un refugio mixto, lo cual permitiría la presencia de la comunidad de Ostional en el área del RNVSO (Salazar y Rojas 2012). Estos últimos autores fundamentan la idea de que el RNVSO se creó posterior (17 de noviembre de 1983) al establecimiento de la comunidad de Ostional como tal (antes de 1950). Para la fecha de creación del refugio, el área ya estaba ocupada y en algunos casos las personas ya contaban con el título de propiedad inscrito en el Registro Público de la Propiedad (Salazar y Rojas 2012). No obstante, la Sala Constitucional declaró que el RNVSO es un bien demanial y es por esto que se debe hacer el desalojo del área y por tanto, solo se deben entregar permisos para protección e investigación. Sin embargo, la misma Sala se contradice, ya que por otro lado acepta otorgar permisos de uso para el turismo dentro del RNVSO. A pesar de ello, debido a que el RNVSO se encuentra en la zona marítima terrestre, es Patrimonio Natural del Estado y por lo tanto corresponde a un refugio estatal, así que los terrenos ocupados dentro del RNVSO deben desalojarse (Salazar y Rojas 2012). EI MINAE y la Unidad Ejecutora del Programa de Regularización entre Catastro y Registro mantienen la tesis de que el RNVSO debe ser regulado como de propiedad mixta. Lo anterior, debido a que el Estado conformó el refugio e incluyó los terrenos debidamente inscritos ante el Registro Público, lo cual denota la intención de clasificar el refugio como mixto, más aún cuando nunca se gestionó por el Estado la recuperación de los terrenos privados (Salazar y Rojas 2012).

Aparentemente, la situación expuesta se aclarará muy pronto pues estaba previsto que a finales de noviembre de 2015 la asamblea legislativa discutiera la "Ley Para La Constitución del Refugio Nacional de Vida Silvestre Ostional en Territorio Costero 
Comunitario". Esta ley tiene por objeto establecer un régimen jurídico especial para el Refugio de Vida Silvestre Ostional. Con esta ley se cambian los usos del suelo a un régimen especial de concesiones. La ley incluye el aprovechamiento razonable y sustentable de los recursos naturales mediante la participación activa de las comunidades y brinda seguridad jurídica a quienes actualmente ocupan terrenos del refugio.

Aspectos sociales. La comunidad de Ostional depende de la extracción de los huevos para su supervivencia. Al parecer, las primeras familias que colonizaron Ostional, a principios de los años 50, dependían económicamente de la agricultura, pesca y ganadería a pequeña escala. Después de la construcción de la carretera de Santa Cruz-Ostional, aumentó la poblacional en la comunidad, por lo que se inició la extracción de huevos de tortuga de manera ilegal como actividad económica (Campbell 1998). La creación del proyecto legal de extracción de huevos de tortuga de Ostional ha hecho que la comunidad dependa de la comercialización de estos huevos.

Para 1992, el 60\% de la población identificaba la extracción de huevos como la actividad económica más importante para sus hogares, mientras que el $31 \%$ identificaba a la agricultura como tal. Para 1997, el $72 \%$ de los hogares identificaban la extracción como la actividad económica principal, mientras que solo un $22 \%$ señalaba a la agricultura (Campbell 1998). No se sabe qué tan significativo es este aumento desde el punto de vista de la población de tortuga lora.

Entre 1992 y 1995, el salario que se le pagaba a los asociados de la ADIO era de aproximadamente US $\$ 10$ a US $\$ 20$ por $12-15$ horas laborales en la extracción y 4 horas extra en otras actividades relacionadas al proyecto. En contraste, se pagaban $\$ 1.25$ por 5 horas laborales en la agricultura o US\$4 por trabajo en la construcción (Campbell 1998). El hecho de que la extracción tenga una remuneración mayor a otras actividades, ha fomentado que las personas basen su economía en la extracción. Muchos de los asociados solo trabajan durante la temporada de arribadas, mientras que el resto del tiempo se dedican a actividades no productivas, lo cual fomenta vicios como el alcoholismo y la drogadicción (García y McHugh 2005).

$\mathrm{Si}$ por aspectos biológicos el proyecto de explotación de huevos tuviera que cerrar, la comunidad no sería capaz de cambiar su actividad económica a corto plazo. Las arribadas podrían desaparecer tan súbitamente como aparecieron. Las tortugas llegan a la playa en donde nacieron que reconocen por un proceso de impronta (Mora y Robinson 1982). Esto sería un catalítico que lleva a la creación de grandes arribadas pues cada vez habría más tortugas que lleguen a la playa donde nacieron. Sin embargo, si en un punto dado la playa no resulta tan segura, o algún otro factor ecológico, la población podría, paulatina o súbitamente, abandonar el comportamiento o la playa de arribada. Esto depende de los mecanismos biológicos que regulan estos aspectos, los cuales se desconocen. No obstante, las arribadas en playa Nancite en el Parque Nacional Santa Rosa han disminuido drásticamente en la últimas décadas, aunque aún no se entienden por completo las interacciones que llevan a la disminución de la población de tortugas en esta área protegida (Cornelius et al. 2007). Una situación como la descrita podría ser un problema para todas las familias que dependen del ingreso económico producto de los huevos de tortuga, ya que las otras ofertas laborales no solo son menos lucrativas y pocas, sino que están disponibles únicamente para cierta parte de la población (García y McHugh 2005).

Cada arribada genera alrededor de $\$ 40,000$ de los que el $70 \%$ se distribuye entre las familias y se usa para la construcción de infraestructura en el poblado. Se ha invertido en el mejoramiento de los servicios de electricidad, el cuidado del agua, la salud, mantenimiento de los caminos y educación. El 30\% restante se invierte en el funcionamiento e infraestructura de la ADIO. Además, parte de los huevos que se colectan son donados a asilos de ancianos y comedores infantiles. La ADIO proporciona beneficios a sus 260 socios-beneficiarios actuales, incluida la asignación de becas de estudios y pensiones para adultos mayores.

Afortunadamente, la comunidad de Ostional no solo recibe beneficios económicos de la actividad de extracción y comercialización sostenible de los huevos, sino que también se beneficia del turismo relacionado con el avistamiento de las tortugas marinas. Hay dos asociaciones comunitarias de guías turísticos que se encargan de guiar a los turistas en la observación de las tortugas y el proceso de desove.

La actividad turística beneficia a la comunidad de Ostional, a los turistas y a las estrategias de conservación de las tortugas marinas. La comunidad recibe beneficios económicos de los servicios que presta el turismo (visitas guiadas, hospedaje y 
alimentación). Los turistas ven las tortugas y aprenden de su biología y además interactúan con todas las expresiones sociales, culturales y económicas de Ostional. Además, debido a la interacción entre la comunidad con los turistas y las tortugas, se genera más conciencia social sobre la importancia de conservar a la especie y los lugares donde se reproducen. Sin embargo, esta actividad debe ser regulada y bien manejada por las autoridades del MINAE y de la ADIO.

El avistamiento de tortugas supuestamente se encuentra regulado para que no afecte 0 altere a las hembras mientras están anidando. Sin embargo, durante este trabajo nuestros grupos ingresaron y salieron del refugio de día y de noche sin restricción ni indicaciones de ningún tipo (tamaños de grupo, uso de luz y otros). Esto se debió quizá a que nuestras visitas no fueron en días de arribada. Sin embargo, la playa de anidación necesita todos los días vigilancia y control, aunque no sean de arribada.

Se han dado casos de descontrol total en el ingreso de turistas a la playa, incluso en días de arribada. El 6 de septiembre de 2015, la playa principal de anidación de Ostional estuvo llena de turistas que interrumpieron el paso de las tortugas, tanto así, que muchas tortugas regresaron al mar sin haber desovado (Delfino 2015, Medina 2015). Este hecho fue denunciado por varios medios de comunicación colectiva (El País, La Nación, La Prensa Libre, Repretel, Tico Times y New York Times). Es claro que hay un vacío sensible en el control y manejo del ingreso de turistas y otras personas al RNVSO. La administración del RNVSO ha asegurado que coordinará con quién corresponda para evitar que se repitan estas situaciones (Delfino 2015, Medina 2015).

La comunidad de Ostional es consciente de que las actividades de extracción y comercialización de los huevos y las actividades turísticas asociadas deben ser realizadas de una forma sostenible. Ellos mantienen una estrecha relación con la naturaleza y prefieren evitar la pavimentación de los caminos para que de esta forma no se altere el ambiente natural de la zona (ADIO et al. 2008). En el 2000, el proyecto fue suspendido, sin embargo, la comunidad siguió limpiando y patrullando la playa (ADIO et al. 2008). Esto muestra el apoyo que la sociedad local brinda al proyecto de aprovechamiento y el interés que tienen por conservar la especie. Estos valores son inculcados a los pobladores desde niños ya que son involucrados en las actividades de protección de las tortugas, ayudan a ahuyentar a los depredadores, liberan crías y limpian las playas.

Según varios argumentos, Ostional aparece como un caso exitoso de aprovechamiento de la fauna silvestre. El proyecto proporciona una alternativa económica sostenible a una comunidad pobre y con muy pocas alternativas de desarrollo, así como fuentes de trabajo (García y McHugh 2005). El proyecto de aprovechamiento de huevos de tortugas de Ostional es un ejemplo de la necesidad de tomar en cuenta a las comunidades relacionadas con proyectos de conservación (Townsend y Rumiz 2004). Gracias al proyecto de explotación comercial de los huevos producto de las arribadas, la comunidad de Ostional ha adquirido el compromiso de proteger las tortugas (García y McHugh 2005).

Argumentación en contra del aprovechamiento. Uno de los argumentos más fuertes en contra del proyecto de extracción de huevos en Ostional es que este justifica la cosecha y aumenta la caza furtiva de tortuga lora y otros huevos de tortugas marinas en Costa Rica. Una evaluación de qué hacer con la cosecha tiene que tomar en cuenta no solo el impacto de la extracción en la población que anida en Ostional, sino también el impacto en la conservación de las tortugas marinas en el resto del país. Sin embargo, esta no es una labor que corresponda, al menos necesariamente y completamente, a la comunidad de Ostional, es una labor de las autoridades correspondientes de los ministerios involucrados. El papel y deber de la comunidad de Ostional es cumplir con los requisitos dictados por los planes quinquenales de explotación y el plan general de manejo del RNVSO así como los lineamientos que la misma ADIO establezca según lo dicte la argumentación científica correspondiente.

El comercio ilegal es uno de los aspectos que más debilita al proyecto de extracción de huevos (Mora 2012). Se argumenta que la existencia de huevos de tortuga legales es lo que permite que la extracción de huevos ilegales en otras playas no se haya podido erradicar (Blanco et al. 2012, Orrego 2014). Sin embargo, no es asunto de Ostional si no hay control efectivo de la extracción de huevos no permitida de otras especies y en otros sitios.

Costa Rica protege las tortugas marinas y ha firmado convenios internacionales sobre esta temática, incluida la Convención Interamericana de Tortugas Marinas. Debido a esto, la explotación de huevos de 
tortuga lora no debería darse. Además, se ha argumentado que el consumo de huevos de tortuga, pone al país en una posición de doble discurso, ya que por un lado se fomenta la conservación y por otro se explota un recurso natural que se encuentra protegido en el resto del mundo. Sin embargo, el uso sostenible de este recurso muestra al mundo la capacidad del país de conservar la biodiversidad bajo los objetivos de la CDB (Convención de Biodiversidad) de proteger el recurso a largo plazo y procurar su utilización racional por las comunidades humanas. Legalmente, la actividad de extracción de huevos en Ostional está amparada bajo el decreto ejecutivo de 1990, la ley № 8436 de Pesca y Acuicultura y Ley $N^{\circ} 8325$ de Protección, Conservación y Recuperación de las Poblaciones de Tortugas Marinas.

El argumento más fuerte en contra del aprovechamiento de los huevos en Ostional es la afectación de la población de tortugas. La UCR ha generado datos que indican que la población de la tortuga lora en Ostional no ha disminuido (Cornelius y Robinson 1985; Cornelius et al. 1991; Chaves y Morera 2003) o que ha aumentado (Chaves 2007, ADIO et al. 2012). Otros estudios indican que la población se ha mantenido constante (Valverde et al. 2013). La conclusión sería que el proyecto de aprovechamiento de huevos no está impactando de forma negativa a la tortuga lora. No obstante, un vacío es la falta de publicaciones científicas de respaldo. Algunas de las referencias bibliográficas citadas antes se refieren a datos de hace ya varios años o no son artículos en revistas científicas. Se necesitan publicaciones científicas actuales que respalden los argumentos a favor de la explotación de huevos de tortuga en Ostional. Los documentos disponibles más recientes y actualizados, aparentemente no apoyan la explotación de huevos tal y como ocurre en este momento (Valverde et al. 2012, Orrego 2014).

Monitoreo de la tortuga lora en Ostional. Es importante entender la interacción entre los procesos históricos y las decisiones políticas y económicas que explican el uso y la tendencia del ecosistema del que la fauna de interés forma parte. Esto se debe anteponer a cualquier planteamiento o medida de aprovechamiento o conservación de la especie (Barbarán 2003).

Las poblaciones de la tortuga lora han disminuido en el mundo (Abreu-Grobois y Plotkin 2008). En Mesoamérica, el decline poblacional ha sido severo debido, en parte, a la explotación ilegal y legal de sus huevos (Cornelius et al. 2007). Las características de su biología, como su extrema fidelidad al nido donde nacieron, así como el arribo predecible y masivo, han hecho a la tortuga lora un blanco fácil para la explotación por los recolectores furtivos de huevos, lo que ha traído como consecuencia la disminución drástica de sus poblaciones en algunas zonas (CONANP 2008). Esto, más el desconocimiento existente acerca de las arribadas y sus mecanismos biológicos, hacen riesgoso cualquier tipo de manejo (Marcovaldi 2001).

Existen grandes diferencias regionales en las estimaciones poblacionales de la tortuga lora. Se ha señalado que el declive neto de esta especie se propicia principalmente por las tendencias de las dos poblaciones que anidan en arribada, una en Escobilla (México) y la otra en Ostional (Abreu-Grobois y Plotkin 2008). Esto podría ser un argumento fuerte en contra de la explotación legal de huevos en Ostional. Sin embargo, la población de hembras que anida en Ostional al menos se ha mantenido igual (Valverde et al. 2012) o incluso ha aumentado (Chaves 2007).

La supuesta evidencia generada por la UCR establece un aumento de las poblaciones, al menos del número de hembras de tortuga lora que anida en Ostional (Chaves 2007), aunque no hay publicaciones científicas recientes con esta información. Valverde et al. (2012) afirmaron que existen grandes fluctuaciones en las poblaciones de tortuga lora, por lo que no se encontró una tendencia poblacional que sustente su explotación en Ostional. Aun así, estos autores concluyeron que la población de la tortuga lora en Ostional está estable. Valverde et al. (2012) señalaron que podría haber ocurrido un decremento en la población durante las dos últimas décadas lo cual no ha sido posible detectar. Sin embargo, si no se conoce el tamaño poblacional, no se sabe si la explotación de huevos o las actividades de manejo en la playa (quema de madera y siembra de árboles y la remoción de troncos, hierba y vegetación rastrera) están impactando a la población positiva o negativamente.

El monitoreo de las tortugas en Ostional ha sido ejecutado por los investigadores de la Estación Biológica Douglas Robinson de la UCR quienes han registrado a diario el número de tortugas que ingresan a la playa, el número de nidos y la destrucción de los mismos por factores antrópicos, animales 0 ambientales. También miden la duración de las arribadas y se estima el tamaño poblacional de la especie (ADIO et al. 2012, Orrego 2014). Además, se 
han investigado otros aspectos sobre la biología de la tortuga lora y de las condiciones del hábitat. Entre estos están aspectos relacionados a la tasa de éxito de eclosión de nidos naturales (Alvarado-Ulloa 1985), la viabilidad de los huevos (Chaves 1986), la influencia de la temperatura en la determinación del sexo (Ugalde 1986), la contribución de las tortugas solitarias en el mantenimiento de la población (Castro 1986) y las tasas de eclosión (Chaves 2007).

La Universidad Nacional (UNA) también ha investigado en el sitio, por ejemplo sobre la carga microbiana en la playa (Mo et al. 1992) y la tasa de eclosión en nidos marcados (Araúz-Almengor 1993). Otras instituciones e investigadores se han unido a la tarea de generar información sobre la tortuga lora en Ostional con temas como la temperatura de incubación (Valverde et al. 2010), los mecanismos que determinan las arribadas (Valverde et al. 2012) y aspectos de biología y manejo en general de la especie (Orrego 2014).

El monitoreo de la población de tortugas es central para el aprovechamiento de los huevos y uno de los que ha generado más controversia. Para el monitoreo de la tortuga lora en Ostional se han utilizado tres modelos de abundancia. Dichos modelos difieren en su metodología y objetos de estudio, lo que limita la comparación de los datos (NMFS y USFWS 2014). Este vacío es una barrera para el entendimiento de la dinámica poblacional de la especie y dificulta la medida del impacto de la explotación de los huevos en la población de tortugas. Chaves (2007) señaló que la población de la tortuga lora en Ostional está aumentando, no obstante, Valverde et al. (2012) argumentaron que la población apenas se mantiene constante. Esta debilidad de la base científica para el aprovechamiento supone un riesgo para la especie.

La premisa principal de la ley que permite la explotación de huevos en Ostional no se está cumpliendo. Este es entonces, el vacío más importante en el proyecto y son necesarias las investigaciones pertinentes o las publicaciones científicas de los datos ya recopilados que permitan a los entes responsables tomar las decisiones más adecuadas al respecto. Además, hace falta investigar el estado del ecosistema marino-costero del RNVSO. La mayor parte de los estudios en Ostional se han enfocado en las arribadas. Se han quedado por fuera aspectos del ambiente marino-costero que pueden influir sobre la población de la tortuga lora.

\section{Conclusiones y Recomendaciones}

En Costa Rica existe un ejemplo claro donde el manejo comunitario ha logrado favorecer a la comunidad local y a la vida silvestre, la comunidad de Ostional en el noroeste del país. La extracción de huevos de la tortuga lora (Lepidochelys olivacea) ha logrado mantener la población de tortugas que anidan y han aumentado el número de anidamientos (AbreuGorbois y Plotkin 2008). Se ha estimado una tendencia al aumento anual de hembras por arribada (Valverde et al. 2012, NMFS y USFWS 2014). No solo ha aumentado el número de hembras, sino que ha aumentado también el número de arribadas por año y las hembras utilizan más áreas para anidar aparte de la playa principal de anidación (Chávez 2007). Esto contrasta con el caso de playa Nancite, en el Parque Nacional Santa Rosa, donde se ha encontrado que la población que llega a anidar ahí ha disminuido considerablemente a pesar de estar dentro de un área de protección total (Fonseca et al. 2009). A pesar de esta tendencia, en el 2007 se detectaron más nacimientos de tortugas en Nancite, lo que se achaca a la disminución de la influencia de los factores densodependientes de la playa (NMFS y USFWS 2014).

Del aprovechamiento de huevos de tortuga, la comunidad ha logrado desarrollarse y se ha vuelto autogestora del recurso bajo el soporte técnico y científico de las universidades y el Estado. El ejemplo de Ostional puede ser tomado como base para aplicarlo a otros sitios donde las zonas de pobreza se asocian a las áreas protegidas. La restricción del uso de los recursos y el evitar el manejo conjunto de la vida silvestre son aspectos negativos para las comunidades y un lujo para personas ajenas al sitio. Es necesario incluir a las comunidades y su cultura en el manejo de los recursos naturales, aun en áreas protegidas, que pese a su estatus no están exentas de la explotación ilegal que podría ser regulada desde la comunidad y esta manejar los recursos para su beneficio (Palomo et al. 2014). Se deben analizar las estrategias de conservación, fortalecer el vínculo que existe entre el gobierno, las universidades y las comunidades locales para lograr un manejo comunitario con buen fundamento legal y científico.

El caso de Ostional puede ser considerado como un caso de aprovechamiento de un recurso considerado como renovable y abundante. De cierta manera, se trata de una explotación experimental donde los científicos, los organismos gubernamentales y la misma comunidad obtienen ingresos económicos. 
No obstante, un experimento de prueba y error puede tener consecuencias muy negativas para la población a largo plazo de una especie longeva si no se sabe detener a tiempo. Por lo tanto, el plan de manejo de Ostional y el plan de aprovechamiento de huevos deben ser planes bajo manejo adaptativo (Mora 2003, Williams 2015).

Hacen falta estudios para que el proyecto llegue a operar con bases sólidas y ser sostenible. No se sabe si la extracción de huevos tiene algún efecto sobre la población de tortugas que anida en Ostional. Los parámetros demográficos de la tortuga lora que anida en Ostional están basados en lo que algunos consideran una metodología sesgada e inadecuada o debilidades en la metodología de los conteos (Valverde et al. 1998, Ballestero et al. 1998, Valverde y Gates 1999, Hope 2002).

Los responsables del manejo de los recursos están interesados en conservar las poblaciones que puedan mantener un rendimiento o cosechas sostenidas (Mora 2003). En este contexto, conocer las principales razones de la existencia de las arribadas sería de gran utilidad para el manejo de la tortuga lora. Según ciertos estudios, la temperatura superficial del mar y la presencia de clorofila-a determinan el movimiento de estos quelonios (Polovina et al. 2004, Brenes et al. 2012). No obstante, más investigaciones etológicas deberían haber para esclarecer estas dependencias.

Se recomienda estandarizar los métodos utilizados para el monitoreo de las poblaciones de tortugas marinas que anidan en esta playa, especialmente las técnicas para estimar el tamaño de las arribadas. Esto con el fin de contar con datos que sean comparables a través de los años y de esta forma permitan determinar las fluctuaciones en las poblaciones que anidan allí.

Es recomendable un taller con todos los actores que actúan en la zona: los encargados del control y administración (MINAE), la ejecución (ADIO), la comercialización (INCOPESCA) e investigadores. Esto con el fin de integrar las debilidades y fortalezas dentro y fuera del refugio con las cuales construir una matriz de conflictos y potencialidades. Trabajar en los puntos clave para solucionar los conflictos identificados sería otra de sus funciones. Esto permitirá tener una perspectiva holística del RNVSO y sus interacciones, para generar un plan de manejo y planes de acción que converjan en beneficio del refugio, de la tortuga lora y de la comunidad de Ostional.
No se recomienda incrementar el volumen del aprovechamiento (la extracción) ni expandir la comercialización de huevos a otros sectores. Para ello, se requiere conocer antes el impacto real que el aprovechamiento actual tiene sobre las poblaciones de la tortuga lora.

Las tortugas marinas enfrentan amenazas por diversos factores tales como la pesca incidental, la erosión de las playas de anidación y la extracción ilegal de huevos. Playa Ostional contribuye con un alto número de individuos a las poblaciones de adultos y así ayuda a contrarrestar otras amenazas que las tortugas enfrentan y que son ajenas al aprovechamiento de sus huevos en la zona.

\section{Literatura Citada}

Abreu-Grobois, A. y P.T. Plotkin. 2008. Lepidochelys olivacea. In IUCN 2013. IUCN Red List of Threatened Species. Versión 2013.2. Consultado 05 de junio de 2014. Disponible en: www.iucnredlist.org.

ADIO/EB-UCR/INCOPESCA/RNVSO-SINAC-MINAE

(Asociación de Desarrollo Integral de Ostional, Escuela de Biología de la Universidad de Costa Rica, Instituto Costarricense de Pesca y Agricultura, Refugio Nacional de Vida Silvestre Ostional-Área de Conservación Tempisque-Sistema Nacional de Áreas de Conservación). 2008. Plan quinquenal de aprovechamiento de huevos de tortuga lora en Ostional 2007-2011. Consensuado el 13 de octubre y operativizado el 22 y 23 de noviembre del 2006. 24 p.

ADIO/SINAC/EB-UCR/INCOPESCA/RNVSO-ACT-

SINAC/SNG/SENASA (Asociación de Desarrollo Integral de Ostional, Sistema Nacional de Áreas de Conservación, Escuela de Biología de la Universidad de Costa Rica, Instituto Costarricense de Pesca y Agricultura, Refugio Nacional de Vida Silvestre OstionalÁrea de Conservación Tempisque-Sistema Nacional de Áreas de Conservación, Servicio Nacional de Guardacostas, Servicio Nacional de Salud Animal). 2012. Plan quinquenal de manejo y conservación de tortugas marinas lora del refugio nacional de vida silvestre Ostional. Consensuado entre el 12 y 29 de marzo 2012. Sistema Nacional de Áreas de Conservación de Costa Rica, Costa Rica. 43 p.

Aguirre, A.A., S.C. Gardner, J.C. Marsh, S.G. Delgado, C.J. Limpus y W.J. Nichols. 2006. Hazards associated with the consumption of sea turtle meat and eggs: a review for health care workers and the general public. EcoHealth 3(3):141-153.

Alvarado-Ulloa, M.A. 1985. Tasa de éxito de eclosión de nidos naturales de la tortuga marina Lepidochelys olivacea (Eschscholtz, 1829) en el Refugio Nacional de Fauna Silvestre de Ostional, Guanacaste, Costa Rica. Tesis de Licenciatura. Universidad de Costa Rica. 96 p. 
Araúz-Almengor, M.L. 1993. Tasa de eclosión en nidos marcados de Lepidochelys olivacea en playa Ostional, Refugio Nacional de Vida Silvestre Ostional, Guanacaste, Costa Rica. Tesis de Maestría. Universidad Nacional de Costa Rica. 80 p.

Asamblea Legislativa de la República de Costa Rica. 2009. Proyecto de ley. Ley del Refugio Nacional de Vida Silvestre Ostional. Expediente No. 17.512.

Ballestero, J., R.M. Arauz y R. Rojas. 1998. Management, conservation, and sustained use of olive ridley sea turtle eggs (Lepidochelys olivacea) in the Ostional Wildlife Refuge, Costa Rica: an 11 year review. p. 4-5. In AbreuGrobois, F.A., R. Briseno-Duenas, R. Marquez y L. Sarti. Proceedings of the Eighteenth International Sea Turtle Symposium. U.S. Dept. of Commerce. NOAA Technical Memorandum NMFS-SEFSC-436.

Barbarán, F.R. 2003. Evaluación de sostenibilidad del uso comercial de la fauna Chaqueña: dimensiones económica, social e institucional. p. 67-98. In M.T. Becerra (Ed.). Lineamientos para el manejo sostenible de sistemas de aprovechamiento de recursos naturales in situ. Instituto de Investigación de Recursos Biológicos Alexander von Humboldt. Bogotá, Colombia.

Becerra, M.T. (Ed.). 2003. Lineamientos para el manejo sostenible de sistemas de aprovechamiento de recursos naturales in situ. Instituto de Investigación de Recursos Biológicos Alexander von Humboldt. Bogotá, Colombia. $186 \mathrm{p}$.

Bernardo, J. y P.T. Plotkin. 2007. An evolutionary perspective on the Arribada phenomenon and reproductive behavioral polymorphism of olive ridley sea turtles (Lepidochelys olivacea). p. 59-88. In Plotkin, P.T. Biology and Conservation of Ridley Sea Turtles. Johns Hopkins University Press, Baltimore, MD.

Blanco, G.S., S.J. Morreale, R. Piedra, W. Montes, F.V. Paladino y J.R. Spotila. 2012. Reproductive output and ultrasonography of an endangered population of East Pacific green turtles. Journal of Wildlife Management 76(4):841-846.

Brenes, C.L., A. Márquez, W. Quirós y R.B. Morera. 2012. Rasgos hidrográficos y batimétricos del Refugio Nacional de Vida Silvestre Ostional y áreas adyacentes, Guanacaste, Costa Rica. Revista Ciencias Marinas y Costeras 4(1):145-156.

Burger, J. y M. Gochfeld. 2013. Wood storks (Mycteria americana) prey on eggs and hatchlings of olive ridley sea turtles (Lepidochelys olivacea) at Ostional, Costa Rica. Waterbirds 36(3):358-363.

Campbell, L.M. 1998. Use them or lose them? Conservation and the consumptive use of marine turtle eggs at Ostional, Costa Rica. Environmental Conservation 25(4):305-319.

Campos, E., H. Bolaños, M.T. Acuña, G. Díaz, M.C. Matamoros, H. Raventós, L.M. Sánchez, O. Sánchez, C. Barquero y Red Nacional de Laboratorios para Cólera, Costa Rica. 1996. Vibrio mimicus Diarrhea following Ingestion of Raw Turtle Eggs. Applied and Environmental Microbiology 62(4):1141-1144.
Castro, J.C. 1986. Contribución de las tortugas lora solitarias Lepidochelys olivacea (Eschscholtz) en el mantenimiento de las poblaciones de ésta especie. Práctica dirigida para el grado de Licenciatura. Universidad de Costa Rica. 63 p.

Castro-González, M. I. y R.F. Pérez-Gil. 2011. Composición química del huevo de tortuga golfina Lepidochelys olivacea (Testudines: Cheloniidae) y su potencial como recurso alimenticio. Revista de Biología Tropical 59(4):1729-1742.

Chaves, A. 1986. Viabilidad de los huevos de la tortuga marina Lepidochelys olivacea (Eschscholtz) en Playa Ostional, Guanacaste, Costa Rica. Tesis de Licenciatura. Universidad de Costa Rica. 43 p.

Chaves, G. 2007. Tendencia poblacional y éxito de eclosión de las anidaciones masivas de tortugas lora (Lepidochelys olivacea) en el Refugio Nacional de Vida Silvestre de Ostional, Guanacaste. Tesis de Maestría. Universidad de Costa Rica. 81 p.

Chaves, G. y R. Morera. 2003. Plan de manejo para la utilización racional, manejo y conservación de los huevos de la tortuga marina lora (Lepidochelys olivacea) en el Refugio Nacional de Vida Silvestre Ostional, Santa Cruz, Guanacaste, Costa Rica. Informe 2003. Universidad de Costa Rica. 33 p.

CONANP (Comisión Nacional de Áreas Naturales Protegidas). 2008. Programa de Monitoreo de la Tortuga Golfina (Lepidochelys olivacea) en el Parque Nacional Lagunas de Chacahua. Comisión Nacional de Áreas Naturales Protegidas de México. Consultado 29 de febrero de $2015 . \quad$ Disponible en: http://www.conanp.gob.mx/acciones/fichas/chacahua/

Cornelius, S.E., M.A. Ulloa, J.C. Castro, M. Mata del Valle y D.C. Robinson. 1991. Management of olive ridley sea turtles (Lepidochelys olivacea) nesting at Playas Nancite and Ostional, Costa Rica. p. 111-135. In J.G. Robinson y K.H. Redford (Eds.). Neotropical Wildlife Use and Conservation. The University of Chicago Press, Chicago.

Cornelius, S.E., R. Arauz, J. Fretey, M.H. Godfrey, R. Marquez y K. Shanker. 2007. Effect of land-based harvest of Lepidochelys. p. 231-251. In P.T. Plotkin (Ed.). Biology and Conservation of Ridley Sea Turtles. The Johns Hopkins University Press. Baltimore, MD.

Cornelius, S.E. y D.C. Robinson. 1985. Abundance, distribution and movements of olive ridley sea turtles in Costa Rica. NFWS Report 5. 54 p.

Delfino, D. 2015. Ostional: perspectiva y contexto: Más allá de la "foto pal Face" y el hate automático. Consultado 22 de septiembre de 2015. Disponible en: https://medium.com/@delfinomachin/ostionalperspectiva-y-contexto-95c4a7e37efa\#.180uthqiz.

Eckert, K.L. y F.A. Abreu Grobois (eds.). 2001. Proceedings of the Regional Meeting: "Marine Turtle Conservation in the Wider Caribbean Region: A Dialogue for Effective Regional Management," Santo Domingo, 16-18 November 1999. WIDECAST, IUCN-MTSG, WWF, and UNEP-CEP. $154 \mathrm{p}$. 
Eckert, K.L., K.A. Bjorndal, F.A. Abreu-Grobois y M. Donnelly. 2000. (Traducción al español). Técnicas de Investigación y Manejo para la Conservación de las Tortugas Marinas. Grupo especialista en Tortugas Marinas. Unión Internacional para la Conservación de la Naturaleza y Comisión de Supervivencia de Especies, Publicación No4. 270 p.

Fonseca, L.G., G.A. Murillo, L. Guadamuz, R.M. Spinola y R.A. Valverde. 2009. Downward but stable trend in the abundance of arribada olive ridley sea turtles (Lepidochelys olivacea) at Nancite Beach, Costa Rica (1971-2007). Chelonian Conservation and Biology 8(1):19-27.

García-Díaz, E.G. y A. McHugh. 2005. Ostional: Comunidad modelo en áreas silvestres protegidas. Herencia 18(2):47-58.

Hildebrand, H.H. 1963. Hallazgo del área de anidación de la Tortuga marina "lora" Lepidochelys kempi (Garman), en la costa occidental del Golfo de México. Ciencia México $22(4): 105-112$.

Hope, R. A. 2002. Harvesting, conservation and poverty: the economics of olive ridley eggs exploitation. Environmental Conservation 29(3):375-384.

Kinne, O. (ed.). 1985. Diseases of Marine Animals Vol. IV, Hamburg, Germany: Biologische Anstalt Helgoland. 541 p.

Madden, D., J. Ballestero, C. Calvo, R. Carlson, E. Christians y E. Madden. 2008. Sea turtle nesting as a process influencing a sandy beach ecosystem. Biotropica 40(6):758-765.

Marcovaldi, M.A. 2001. Status and Distribution of the Olive Ridley Turtle, Lepidochelys olivacea, in the Western Atlantic Ocean. p. 16-18. In K.L Eckert y F.A. AbreuGrobois. Proceedings of the Regional Meeting, Marine Turtle Conservation in the Wider Caribbean Region: A Dialogue for Effective Regional Management. WIDECAST, IUCN-MTSG, WWF, and UNEP-CEP.

Medina, M. 2015. Turistas impiden a tortugas marinas desovar en una reserva natural de Costa Rica. Consultado 5 de octubre de 2015. Disponible en: http://ecoinventos.com/turistas-impiden-a-tortugasdesovar-costa-rica/\#ixzz3mNXQBj00.

Mo, C.L., M. Caballero e I. Salas. 1992. Microorganism infection of olive ridley eggs. p. 81-84. In J.I. Richardson y T.H. Richardson (compilers), Proceedings of the Twelfth Annual Workshop on Sea Turtle Biology and Conservation; NOAA Technical Memorandum NMFSSEFSC-361.

Mora, D. 2012. Ambientalistas piden al MINAE que estudie sistema de comercio de huevos de tortuga en Ostional. Consultado 3 de junio de 2014. Disponible en: http://unw.teletica.com/Noticias/19588-Ambientalistas-piden-alMINAE-que-estudie-sistema-de-comercio-de-huevos-de-tortuga-enOstional.note.aspx.
Mora, J.M. 2003. Evaluación y seguimiento para manejo y conservación de fauna silvestre. p. 43-66. In M.T. Becerra (Ed.). Lineamientos para el manejo sostenible de sistemas de aprovechamiento de recursos naturales in situ. Instituto de Investigación de Recursos Biológicos Alexander von Humboldt. Bogotá, Colombia.

Mora, J.M. y D.C. Robinson. 1982. Discovery of a blind olive ridley turtle (Lepidochelys olivacea) nesting at playa Ostional, Costa Rica. Revista de Biología Tropical 30(2):178-179.

Mora, J. M. y D.C. Robinson. 1984. Predation of sea turtle eggs (Lepidochelys) by the snake Loxocemus bicolor Cope. Revista de Biología Tropical 32(1):161-162.

NMFS y USFWS (National Marine Fisheries Service, U.S. Fish and Wildlife Service). 2014. Olive Ridley sea turtle (Lepidochelys olivacea) 5-year review: summary and evaluation. National Marine Fisheries Service, office of protected resources, Silver Spring, Maryland y U.S. Fish and Wildlife Service, Southeast region, Jacksonville Ecological Services field office, Jacksonville, Florida. 87 $\mathrm{p}$.

Orrego, C.M. 2014. Biology and Management of Olive Ridley Turtles (Lepidochelys olivacea) in Central America. Tesis Doctor en Filosofía. Universidad de Drexel. 230 p.

Palomo, I, C. Montes, B. Martín-López, J.A. González, M. García-Llorente, P. Alcorlo y M.R. García Mora. 2014. Incorporating the social-ecological approach in protected areas in the Anthropocene. BioScience 64(3):181-191.

Pereira-Zamora, M., F. Hernández-Chavarría y E. WongGonzález. 2007. Penetración de Vibrio mimicus a través de la cáscara del huevo de tortuga lora (Lepidochelys olivacea). Agronomía Mesoamericana 18(2):247-254.

Polovina, J.J., G.H. Balazs, E.A. Howell, D.M. Parker, M.P. Seki y P.H. Dutton. 2004. Forage and migration habitat of loggerhead (Caretta caretta) and olive ridley (Lepidochelys olivacea) sea turtles in the central North Pacific Ocean. Fisheries Oceanography 13(1):36-51.

Pritchard, P.C H. y P.T. Plotkin. 1995. Olive ridley sea turtle, Lepidochelys olivacea. p. 123-139. In Plotkin, P.T. National Marine Fisheries Service and U.S. Fishand Wildlife Service Status Reviews for Sea Turtles Listed under the Endangered Species Act of 1973. National Marine Fisheries Service, Silver Spring, Maryland.

Salazar, F.D. y P. Rojas. 2012. La situación actual del Refugio Nacional de Vida Silvestre Ostional a la luz del Programa de Regularización entre el Catastro y Registro. Tesis de Licenciatura. Universidad de Costa Rica. 277 p.

Santoro, M.G. Hernández y A.F. García. 2008. Potential Bacterial Pathogens Carried by Nesting Leatherback Turtles (Dermochelys coriacea) in Costa Rica. Chelonian Conservation and Biology 7(1):104-108. 
SINAC (Sistema Nacional de Áreas de Conservación). 2014. Refugio de Vida Silvestre Ostional. Consultado 02 de junio del 2014. Disponible en línea http://www.sinac.go.cr/AC/ACT/RVSOstional/Paginas/d efault.aspx.

Townsend, W.R y D.I. Rumiz 2004. Reflexiones sobre la posibilidad de manejo de fauna silvestre en las tierras bajas de Bolivia: experiencias comunitarias. Revista Boliviana de Ecología y Conservación Ambiental 16:6172.

Ugalde, A. 1986. La temperatura como factor determinante del sexo en la tortuga lora Lepidochelys olivacea Eschscholtz. Tesis de Licenciatura. Universidad de Costa Rica. 60 p.

Valverde, R.A. y C.E. Gates. 1999. Population survey on mass nesting beaches. p. 56. In Eckert, K.L., K. Bjorndal, F.A. Abreu-Grobois y M. Donnelly. Research and management techniques for the conservation of sea turtles. IUCN/SSC Marine Turtle Specialist Group Publication No4.

Valverde. R.A., S.E. Cornelius y C.L. Mo. 1998. Decline of the olive ridley turtle (Lepidochelys olivacea) nesting assemblage at Playa Nancite, Santa Rosa National Park, Costa Rica. Chelonian Conservation and Biology 3(1):58-63.
Valverde, R.A., M.C. Orrego, T.M. Tordoir, M.F. Gómez, S.D. Solís, A.R. Hernández, B.G. Gómez, S.L. Brenes, P.J. Baltodano, G.L. Fonseca y R.J. Spotila. 2012. Olive Ridley Mass Nesting Ecology and Egg Harvest at Ostional Beach, Costa Rica. Chelonian Conservation and Biology 11(1):1-11.

Valverde, R.A., S. Wingard, F. Gómez, T.M. Tordoir y C.M. Orrego. 2010. Field lethal incubation temperature of olive ridley sea turtle Lepidochelys olivacea embryos at a mass nesting rookery. Endangered Species Research 12(1):77-86.

Warwick, C., P.C. Arena y C. Steedman. 2013. Health implications associated with exposure to farmed and wild sea turtles. Journal of the Royal Society of Medicine Short Reports 4(1):8.

Williams, B.K. 2015. Integrating external and internal learning in resource management. Journal of Wildlife Management 79(1):148-155.

Recibido para publicación el 3 de diciembre del 2015.

Aceptado para publicación el 4 de febrero del 2016. 